\title{
Epidemiologic studies of the human microbiome and cancer
}

\author{
Emily Vogtmann ${ }^{1}$ and James J Goedert ${ }^{\star}, 2$ \\ ${ }^{1}$ Nutritional Epidemiology Branch, Division of Cancer Epidemiology and Genetics, National Cancer Institute, National Institutes of \\ Health, 9609 Medical Center Drive, Bethesda, MD 20892, USA and ${ }^{2}$ Infections and Immunoepidemiology Branch, Division of \\ Cancer Epidemiology and Genetics, National Cancer Institute, National Institutes of Health, 9609 Medical Center Drive, Bethesda, \\ MD 20892, USA
}

The human microbiome, which includes the collective genome of all bacteria, archaea, fungi, protists, and viruses found in and on the human body, is altered in many diseases and may substantially affect cancer risk. Previously detected associations of individual bacteria (e.g., Helicobacter pylori), periodontal disease, and inflammation with specific cancers have motivated studies considering the association between the human microbiome and cancer risk. This short review summarises microbiome research, focusing on published epidemiological associations with gastric, oesophageal, hepatobiliary, pancreatic, lung, colorectal, and other cancers. Large, prospective studies of the microbiome that employ multidisciplinary laboratory and analysis methods, as well as rigorous validation of case status, are likely to yield translational opportunities to reduce cancer morbidity and mortality by improving prevention, screening, and treatment.

Human microbiome research has garnered substantial attention, both by scientists and the media. The human microbiome refers to the collective genome of all bacteria, archaea, fungi, protists, and viruses residing in and on the human body. Made feasible by high throughput, next-generation deep sequencing of DNA, as well as expanding computational and bioinformatics support, the microbiome is a conceptual quantum leap from detection and identification of individual microbes to characterisation of entire microbial communities, including both pathogenic and commensal microbes that have not yet been cultured or otherwise detected. Differences among individuals in our co-dependent relationship with the microbiota is postulated to modulate susceptibility to many malignancies via several pathways, including nutrition, detoxification, metabolism, hormonal homeostasis, immune tolerance, and especially inflammation (Sears and Pardoll, 2011; Zhu et al, 2013; Sheflin et al, 2014). Figure 1 presents a schema and analytic approaches for microbiome studies, including both shotgun metagenomics (i.e., sequencing all DNA in a sample) and simpler profiling with $16 \mathrm{~S}$ rRNA gene amplicons of prokaryotes (i.e., bacteria and archaea). For a more comprehensive review of microbiome studies, see (Morgan and Huttenhower, 2012).

The first phase of the Human Microbiome Project (HMP) helped characterise the microbiome of numerous body sites within healthy adults in the United States (The Human Microbiome Project Consortium, 2012); and the second phase, considering the impact of the microbiome on specific health conditions, is underway. Similarly, the Metagenomics of the Human Intestinal Tract (MetaHIT) was established to catalogue the human gut microbiome of Europeans and to identify associations with metabolic and inflammatory bowel diseases (Qin et al, 2010). An altered microbiome linked to a disease has been termed dysbiosis, potentially originating with an 'alpha bug' that exploits an ecologic niche (e.g., porphyromonas gingivalis and periodontal disease) which then induces both inflammation and compensatory responses in the commensal microbial community (Sears and Pardoll, 2011). Dysbiosis-related inflammation, as well as generation of chemical carcinogens including acetaldehyde and $N$-nitroso compounds, are among several mechanisms that have been proposed through which the microbiota may have a role in carcinogenesis (Sears and Pardoll, 2011; Zhu et al, 2013; Sheflin et al, 2014).

In this short review, we will summarise published epidemiological studies of the human microbiome and the risk of specific cancers. These studies of the human microbiome and cancer generally have used three sample types: oral, faecal (as a representation of the distal gut), and tissue samples. This review

*Correspondence: Dr JJ Goedert; E-mail: goedertj@mail.nih.gov

Received 3 September 2015; revised 30 October 2015; accepted 10 November 2015; published online 5 January 2016 


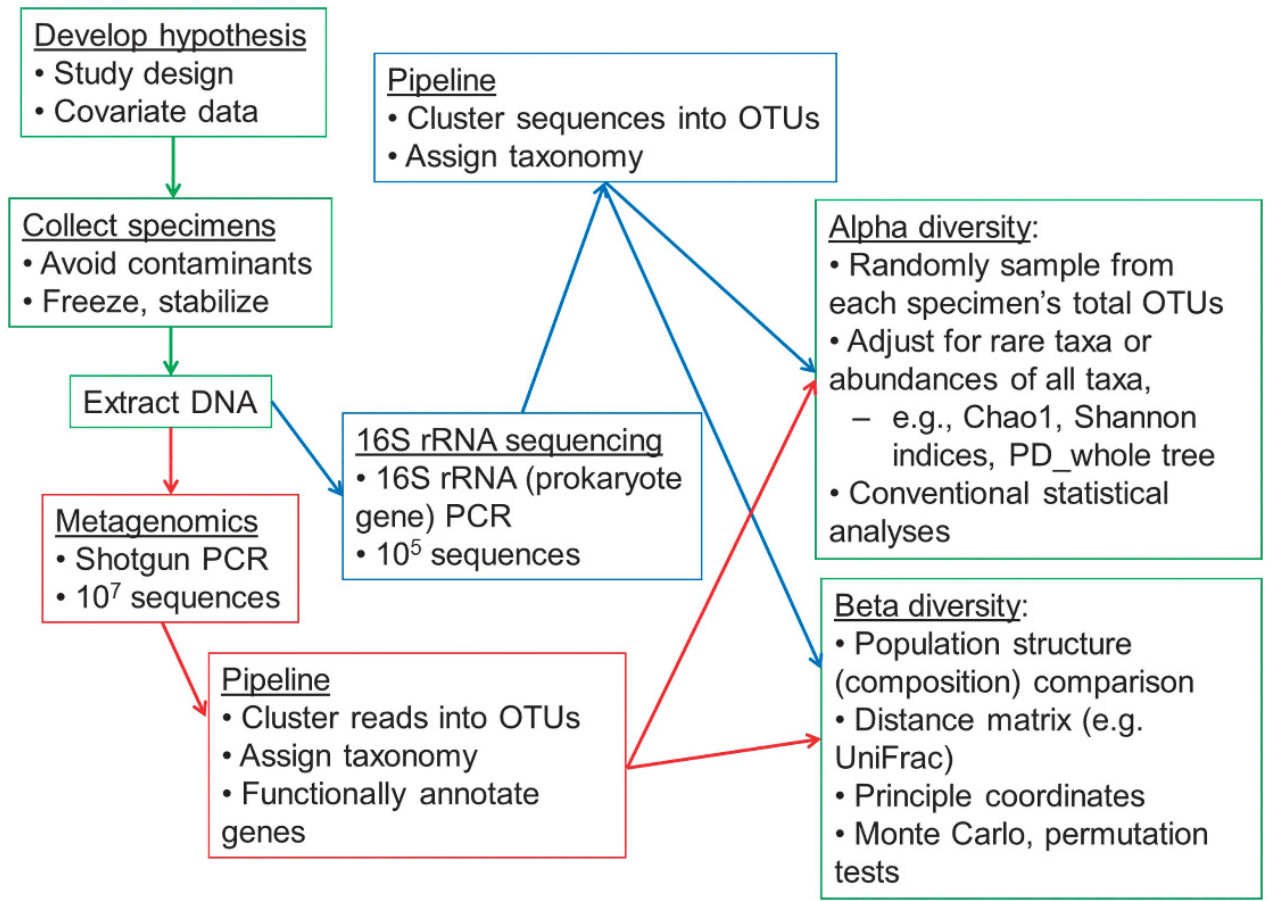

Figure 1. Schema for a human microbiome study. To conduct a human microbiome study, it is important to develop a well-formulated hypothesis, apply a valid study design, and collect requisite covariate data. Relevant specimens (e.g., oral, faecal, tissue, or other applicable samples) should be collected and promptly frozen or chemically stabilised. Once the DNA is extracted, currently there are two typical methods for sequencing: 16S rRNA gene sequencing (in blue) or shotgun metagenomic sequencing (in red). To date, most epidemiologic-scale studies profile the microbiome by amplifying and sequencing only the prokaryote $16 \mathrm{~S}$ rRNA gene. Once the $16 \mathrm{~S}$ rRNA sequencing is completed, the data are often processed using various publically available tools that are used to cluster the sequences into operational taxonomic units (OTUs) and to assign taxonomy using public sequence databases. For shotgun metagenomic sequencing, the DNA is sheared and then all the fragments are sequenced. From this type of data a variety of bioinformatic processing can be conducted, but often the short reads are used to cluster OTUs and assign taxonomy, similar to $16 \mathrm{~S}$, but also to determine the functional capabilities of the genes present by mapping the reads to public gene databases. For both 16S rRNA and shotgun metagenomic sequencing, study participants can be compared by alpha diversity (i.e., within participant diversity) and beta diversity (i.e., between participant diversity) metrics. For alpha diversity, conventional statistical methods are often used. Typically, random sampling for a standardised number of OTUs (i.e., rarefaction) is conducted to minimise bias due to amplification or sampling efficiency and then analyses include adjustment for taxa abundances in order to minimise influence of rare taxa. For associations with the entire microbial community, beta diversity analyses are based on a matrix of distances between all pairs of specimens, followed by principle coordinate analysis and higher level statistics.

will focus primarily on studies including oral or faecal samples, but there is a growing literature, particularly for colorectal cancer (CRC), with informative reports on the microbiome and the tumour microenvironment. The oral and faecal microbiomes differ greatly from each other and may represent separate roles in the carcinogenesis process. In data from the HMP, faecal samples had distinctly different dominant bacterial taxa compared with oral samples. Taxa in the phylum Bacteroidetes predominated in faeces, whereas taxa in the phylum Firmicutes predominated in saliva and oropharyngeal swabs (Segata et al, 2012). This review will also focus exclusively on studies of bacteria and archaea, since there are few, if any, cancer epidemiology studies of other members of the microbiota, with the exception oncogenic viruses, which are outside the scope of this review.

\section{GASTRIC AND OESOPHAGEAL CANCERS}

Gastric cancer has been the model of bacterially associated cancer, as infection with Helicobacter pylori is categorised as a class I carcinogen by the International Agency for Research on Cancer (International Agency for Research on Cancer, 1994). Infection with $H$. pylori generates a strong host immune response, resulting in gastric inflammation, achlorhydria, and epithelial atrophy and dysplasia (Blaser and Atherton, 2004). In prospective epidemio logic studies, $H$. pylori infection has been associated with over a two-fold increased risk of gastric cancer overall (Helicobacter and Cancer Collaborative Group, 2001). Conversely, H. pylori has been associated with a lower risk of oesophageal adenocarcinoma (Islami and Kamangar, 2008), perhaps due to reduced acid reflux and epithelial damage of those tissues.

Only a few studies have looked at the community of bacteria and gastric cancer, and these studies have primary used gastric tissue (Brawner et al, 2014). For example, in a study gastric mucosal tissues from 11 cases of non-cardia gastric cancer, 10 cases of intestinal metaplasia, and 10 cases of chronic gastritis, increasing alpha diversity (i.e., number of different taxa) was observed with increasing disease severity (i.e., gastritis patients had the lowest diversity and gastric cancer cases had the highest alpha diversity) (Eun et al, 2014). However, a comprehensive study including oral, faecal, and gastric tissue samples for microbial assessment will be important to determine the integrated effect of the human microbiome on gastric cancer risk.

Also in the stomach, some $90 \%$ of low-grade lymphomas of the mucosa-associated lymphoid tissue (MALT) appear to be driven by $H$. pylori, and remarkably, eradication of the $H$. pylori infection generally leads to regression of the lymphoma (Wotherspoon et al, 1993). Gastric MALT lymphoma results from chromosomal translocations in $\mathrm{B}$ cells, perhaps due to direct effects of translocated H. pylori CagA protein in these cells (Lin et al, 2010), as well as to direct and indirect immunologic responses 
against $H$. pylori that drive B-cell proliferation (Du and Isaccson, 2002). Regression of lymphoma in $H$. pylori-negative cases who are treated with antibiotics raises the possibility that other bacteria might contribute to gastric MALT lymphomas (Asano et al, 2015). To our knowledge, there have been no epidemiologic studies of the microbial community and MALT lymphomas.

Tooth loss is associated with increased oesophageal cancer risk (Abnet et al, 2001). Most studies published thus far of the microbiome and oesophageal cancer have compared the microbiome of oesophageal tissues, and few distinguish by histology (i.e., adenocarcinoma $v s$ squamous cell carcinoma) that have distinct risk profiles (Yang et al, 2014). A study of gastric corpus tissues sampled from 19 oesophageal squamous cell carcinoma cases, 18 oesophageal squamous dysplasia cases, and 37 age- and sex-matched healthy controls in Iran did not detect overall significant alpha diversity differences between the cases and controls, but models of beta diversity (i.e., between subject community composition) detected statistically significant differences $(P<0.02)$ between matched cases and controls (Nasrollahzadeh et al, 2015). Another study in China evaluated the upper gastrointestinal microbiota using a microarray chip (HOMIM) and samples collected from a balloon device that obtained cells from the stomach, oesophagus, and saliva. Comparing 142 subjects with histologically diagnosed oesophageal squamous dysplasia with 191 without oesophageal squamous dysplasia, the odds of this pre-cancerous lesion was 0.74 (95\% CI: $0.58,0.95)$ per one s.d. increase in the number of detected bacterial genera, which suggests that increased alpha diversity is associated with decreased odds of oesophageal squamous dysplasia. Oesophageal squamous dysplasia also was associated with an altered composition (i.e., beta diversity) of the upper gastrointestinal microbial population (Yu et al, 2014).

\section{HEPATOBILIARY CANCERS}

A recent update of evidence for the carcinogenicity of $H$. pylori provided some data that may suggest an association with hepatocellular carcinoma (HCC) and cholangiocarcinoma, but with numerous concerns about small sample sizes and differing control groups (International Agency for Research on Cancer, 2012). A prospective study in Finland with prediagnostic sera found that antibodies against $H$. pylori-related antigens predicted a non-significant increase in HCC (OR 1.20; 95\% CI: $0.42,3.45)$ and a significant increase in biliary tract cancers (OR 5.47; $95 \%$ CI: 1.17, 25.65) (Murphy et al, 2014). A meta-analysis of 10 studies found that detection of various Helicobacter spp. with various methods also was associated with biliary tract cancer compared with benign biliary disease (pooled OR 3.20; 95\% CI: 2.15, 4.77) (Zhou et al, 2013). In addition, risk for gallbladder cancer has been linked to Salmonella typhi infection. In a meta-analysis of 17 studies, a pooled risk ratio (RR) of 4.28 (95\% CI: 1.84, 9.96) was calculated for the association between $S$. typhi and gallbladder cancer, although only two of the included studies were from cohorts and many of the studies included the entire biliary tract (Nagaraja and Eslick, 2014). Oral and faecal microbiome studies of hepatobiliary malignancies have yet to be published, and comprehensive assessment of the bile microbiome with nextgeneration sequencing methods has thus far been unsuccessful (unpublished observations).

\section{PANCREATIC CANCER}

Risk of pancreatic cancer is increased for people with tooth loss or periodontal disease (Meyer et al, 2008), pointing to a possible contribution of the oral microbiome to this lethal malignancy. However, research on a direct association between the oral microbiome and pancreatic cancer risk has thus far been limited. In a small study of salivary DNA from 10 pancreatic cancer cases and 10 healthy controls, $16(3.9 \%)$ of 410 bacterial taxa present in the HOMIM were significantly associated with case status, although only 6 out of the 16 taxa were confirmed using qPCR. In an independent validation set of saliva specimens from 28 pancreatic cancer cases and 28 healthy controls, out of the 6 confirmed taxa, only Neisseria elongata and Streptococcus mitis were validated as biomarkers for discrimination of pancreatic cancer cases from healthy controls using PCR (Farrell et al, 2012).

A seroepidemiologic case-control approach was used by investigators in the European Prospective Investigation into Cancer cohort. Using an immunoblot array, antibodies were detected and quantified against 25 oral bacterial strains in sera from 405 pancreatic cancer cases and 416 controls, matched on study centre, sex, age at blood collection, date and time of blood collection, fasting and for women, exogenous hormone use. The major finding was that the $6 \%$ of participants with a concentration greater than $200 \mathrm{ng} \mathrm{ml}^{-1}$ of antibodies against $P$. gingivalis ATCC 53978 , a pathogen known to contribute to periodontal disease, had 2.14 times the risk of pancreatic cancer (95\% CI: 1.05, 4.36) compared with those with undetectable or lower levels. Conversely, high antibody titres against commensal oral bacteria, which are generally considered non-pathogenic, were associated with a decreased risk of pancreatic cancer (OR 0.55; 95\% CI: 0.36, 0.83) (Michaud et al, 2013). Antibody titres to oral bacteria depend on numerous individual-specific factors, such as the ability to mount an immunological response, so direct characterisation of the oral microbiota will be needed for better insight into the association with pancreatic cancer.

\section{LUNG CANCER}

Consistent with many of the cancers noted above, periodontal disease has also been associated with lung cancer risk (Meyer et al, 2008), which suggests an association between the oral microbiome and lung cancer risk. In support of this association, links between lung infections and lung cancer have also been observed in previous studies. There is fairly strong evidence that pulmonary Mycobacterium tuberculosis (TB) infection is associated with risk of lung cancer, even among never smokers. In a meta-analysis of 30 studies, the pooled RR for the association between TB and lung cancer was 1.76 (95\% CI: 1.49, 2.08) (Brenner et al, 2011). Similarly, pneumonia that is often caused by various non-TB bacterial and other pathogens is associated with increased risk of lung cancer. In a meta-analysis of 22 studies, the pooled RR for pneumonia and lung cancer was 1.43 (95\% CI: 1.22, 1.68) (Brenner et al, 2011). Of note, however, the majority of the included studies in the meta-analysis were from case-control designs.

One small study investigated the association between lung cancer and the microbiome found in oral wash and sputum samples. The study included only eight never-smoking female lung cancer cases and eight never-smoking female controls in China (Hosgood et al, 2014). From the 16S rRNA gene sequencing results, community diversity in the oral wash samples was not statistically different between cases and controls, but there was an indication that lung cancer cases had a decreased relative abundance of the phyla Spirochaetae and Bacteroidetes and an increased relative abundance of Firmicutes in the oral wash. Community diversity in the sputum samples was marginally lower in the lung cancer cases. Lung cancer cases had a decreased relative abundance of the phyla Spirochaetae and Synergistetes in the sputum samples. 


\section{COLORECTAL CANCER AND COLORECTAL ADENOMA}

Seven studies, each in a different population, have compared the faecal microbiome with CRC (Ahn et al, 2013; Weir et al, 2013; Wu et al, 2013; Zackular et al, 2014; Zeller et al, 2014; Feng et al, 2015; Yu et al, 2015). In general, community (i.e., alpha) diversity was similar or slightly reduced in cases, with the exception of one study that found higher gene and genus richness in the CRC cases (Feng et al, 2015). In contrast, all but the smallest study (Weir et al, 2013) reported case-control differences in the composition of the microbiome that was commonly driven by high prevalence and levels of Fusobacterium and Porphyromonas, as well as lower levels of Ruminococcus in faeces from CRC cases. In one study, Fusobacterium was detected in $31.9 \%$ of CRC cases $v$ s $11.7 \%$ of controls (OR 4.11; 95\% CI: 1.62, 10.47) and Porphyromonas was detected in $27.7 \%$ of cases vs $7.5 \%$ of controls (OR 5.17; 95\% CI: 1.75, 15.25) (Ahn et al, 2013). These studies did not agree on the many other taxa that differed between cases and controls, perhaps because of the differences in assay methods, overall population, case-control matching, diet or medication use, tumour stage, or unrecognised confounders.

Five studies have assessed the association between the faecal microbiome and colorectal adenoma (CRA) (Chen et al, 2013; Zackular et al, 2014; Zeller et al, 2014; Feng et al, 2015; Goedert et al, 2015a). Similar to the studies of CRC, community diversity was generally similar in faeces from CRA cases and controls, but some differences were detected for microbial composition. However, the particular taxa associated with CRA case status varied by study and were less consistent than in the CRC studies. Only single studies detected associations with Fusobacterium (Feng et al, 2015) or Porphyromonadaceae (Zackular et al, 2014). The lack of consistent associations for CRA suggests that detection of certain bacteria in faeces might facilitate detection of CRC but not high-risk CRA. The inconsistency also suggests that aggressive periodontal pathogens may only contribute late in the neoplastic process, or that they merely exploit and replicate effectively in the malignant tissue.

\section{OTHER MALIGNANCIES}

There are indications of microbial associations with a few other cancer sites. As with cancers of the stomach, oesophagus, pancreas, and lung, cancers of the head and neck are associated with tooth loss and periodontal disease. For example, in a meta-analysis of eight studies, the pooled RR for head and neck cancer with periodontal disease was 2.63 (95\% CI: 1.68, 4.14) (Zeng et al, 2013). Tobacco use and alcohol consumption are also strong risk factors for head and neck cancer (World Cancer Research Fund/American Institute for Cancer Research, 2007), and it has been suggested that microbes may mediate these relationships with head and neck cancer. For example, Neisseria, which frequently inhabits the oral mucosa, has been observed to have high alcohol dehydrogenase activity that converts ethanol to acetaldehyde, a carcinogen (Muto et al, 2000). Specific studies of the oral microbiome with head and neck cancer have predominantly focused on oral cancer, and most of these studies have been tissue based or used older technology (Wang and Ganly, 2014).

Recent work has detected an association between the gut microbiome and breast cancer in 48 postmenopausal women, specifically lower community diversity and altered composition compared with 48 control women from the same population (Goedert et al, 2015b). This association has been hypothesised to relate to effects of the gut microbiota on both enterohepatic circulation of oestrogens and on oestrogen-independent pathways such as inflammation.
Lymphoma risk could be affected by alteration of the microbial population, given the intimate, bidirectional relationship between microbes and host immune responses. Although the microbiome has not yet been comprehensively assessed, cutaneous B-cell non-Hodgkin lymphoma was associated with seropositivity to Borrelia burgdorferi, the agent of Lyme disease, in one of two studies in Scandinavia (Schollkopf et al, 2008; Chang et al, 2012). Chlamydophila psittaci, the agent of psittacosis, has been detected by amplification and sequencing in MALT lymphomas in various non-gastrointestinal organs (Aigelsreiter et al, 2011). In the only epidemiologic study of the microbiome and lymphoma, a small case-control study of twins, 13 survivors of adolescent/young adult Hodgkin lymphoma had significantly lower community diversity in faeces compared with their 13 unaffected co-twin controls (Cozen et al, 2013). The lower diversity may support the hypothesis that a 'hygienic environment' contributes to adolescent/young adult Hodgkin lymphoma, but the difference may also have been a consequence of the malignancy or its treatments.

\section{CONCLUSIONS AND FUTURE PERSPECTIVES}

There is epidemiologic evidence for associations between the human microbiome and cancer, particularly gastric and colorectal cancer. However, epidemiologic studies of this association have thus far been very limited, typically with small sample sizes and cross-sectional designs with single-time sampling. Although case-control studies can provide initial insights into microbial associations with cancer, reverse causation is of great concern. In a case-control study, it is not possible to determine whether the carcinogenic process changes the local environment and creates a new niche for microbes or whether alterations in the microbial population or its functions contribute to carcinogenesis. New studies that incorporate repeated, prospectively collected oral, faecal, tissue, and other samples will be important to elucidate the temporal nature of microbial associations with cancer.

Future studies should also incorporate the study of fungi, protists, and viruses, in addition to bacteria and archaea, to fully characterise the human microbiome and its relationship with cancer risk. In addition, standardised methods for the collection of samples, preparation and handling of samples, and bioinformatic processing of data are needed and work is ongoing in this area (e.g., www.mbqc.org). For a review of the technical challenges and especially the critically important design requirements for epidemiologic and translational microbiome studies, see (Goedert, 2013). Finally, there is a need to explore postulated microbemediated carcinogenic mechanisms through transcriptomics, proteomics, metabolomics, and novel immunologic assays.

Microbiome associations with cancer may differ across many host factors, including sex, age, smoking, alcohol consumption, diet, obesity, physical inactivity, and polymorphisms in major human oncogenes. Explicit consideration of these host factors may yield clear stratification of microbiome associations with the various malignancies. Ultimately, across the identified strata, microbiome associations should be translated into practical applications in order to accelerate the diagnosis of cancer or precancer, to increase efficacy and reduce toxicity of cancer therapy (Wallace et al, 2010; Iida et al, 2013), and ideally to prevent cancer by interrupting a microbial carcinogenic pathway.

\section{ACKNOWLEDGEMENTS}

We thank our many microbiome and epidemiology collaborators for advice and inspiration. This work was supported by the National Cancer Institute Intramural Research Program, ZIA-CP-010214. 


\section{CONFLICT OF INTEREST}

The authors declare no conflict of interest.

\section{REFERENCES}

Abnet CC, Qiao YL, Mark SD, Dong ZW, Taylor PR, Dawsey SM (2001) Prospective study of tooth loss and incident esophageal and gastric cancers in China. Cancer Causes Control 12(9): 847-854.

Ahn J, Sinha R, Pei Z, Dominianni C, Wu J, Shi J, Goedert JJ, Hayes RB, Yang L (2013) Human gut microbiome and risk for colorectal cancer. J Natl Cancer Inst 105(24): 1907-1911.

Aigelsreiter A, Gerlza T, Deutsch AJ, Leitner E, Beham-Schmid C, Beham A, Popper H, Borel N, Pospischil A, Raderer M, Kessler HH, Neumeister P (2011) Chlamydia psittaci infection in nongastrointestinal extranodal MALT lymphomas and their precursor lesions. Am J Clin Pathol 135(1): 70-75.

Asano N, Iijima K, Koike T, Imatani A, Shimosegawa T (2015) Helicobacter pylori-negative gastric mucosa-associated lymphoid tissue lymphomas: a review. World J Gastroenterol 21(26): 8014-8020.

Blaser MJ, Atherton JC (2004) Helicobacter pylori persistence: biology and disease. J Clin Invest 113(3): 321-333.

Brawner KM, Morrow CD, Smith PD (2014) Gastric microbiome and gastric cancer. Cancer J 20(3): 211-216.

Brenner DR, McLaughlin JR, Hung RJ (2011) Previous lung diseases and lung cancer risk: a systematic review and meta-analysis. PloS One 6(3): e17479.

Chang CM, Landgren O, Koshiol J, Bjorkholm M, Love TJ, Kristinsson SY (2012) Borrelia and subsequent risk of solid tumors and hematologic malignancies in Sweden. Int J Cancer 131(9): 2208-2209.

Chen HM, Yu YN, Wang JL, Lin YW, Kong X, Yang CQ, Yang L, Liu ZJ, Yuan YZ, Liu F, Wu JX, Zhong L, Fang DC, Zou W, Fang JY (2013) Decreased dietary fiber intake and structural alteration of gut microbiota in patients with advanced colorectal adenoma. Am J Clin Nutr 97(5): 1044-1052.

Cozen W, Yu G, Gail MH, Ridaura VK, Nathwani BN, Hwang AE, Hamilton AS, Mack TM, Gordon JI, Goedert JJ (2013) Fecal microbiota diversity in survivors of adolescent/young adult Hodgkin lymphoma: a study of twins. Br J Cancer 108(5): 1163-1167.

Du MQ, Isaccson PG (2002) Gastric MALT lymphoma: from aetiology to treatment. Lancet Oncol 3(2): 97-104.

Eun CS, Kim BK, Han DS, Kim SY, Kim KM, Choi BY, Song KS, Kim YS, Kim JF (2014) Differences in gastric mucosal microbiota profiling in patients with chronic gastritis, intestinal metaplasia, and gastric cancer using pyrosequencing methods. Helicobacter 19(6): 407-416.

Farrell JJ, Zhang L, Zhou H, Chia D, Elashoff D, Akin D, Paster BJ, Joshipura K, Wong DT (2012) Variations of oral microbiota are associated with pancreatic diseases including pancreatic cancer. Gut 61(4): 582-588.

Feng Q, Liang S, Jia H, Stadlmayr A, Tang L, Lan Z, Zhang D, Xia H, Xu X, Jie Z, Su L, Li X, Li X, Li J, Xiao L, Huber-Schonauer U, Niederseer D, Xu X, Al-Aama JY, Yang H, Wang J, Kristiansen K, Arumugam M, Tilg H, Datz C, Wang J (2015) Gut microbiome development along the colorectal adenoma-carcinoma sequence. Nat Commun 6: 6528.

Goedert JJ (2013) The microbiota and human health: beyond exploration. Eur $J$ Clin Invest 43(7): 657-659.

Goedert JJ, Gong Y, Hua X, Zhong H, He Y, Peng P, Yu G, Wang W, Ravel J, Shi J, Zheng Y (2015a) Fecal microbiota characteristics of patients with colorectal adenoma detected by screening: a population-based study. EBioMedicine 2(6): 597-603.

Goedert JJ, Jones G, Hua X, Xu X, Yu G, Flores R, Falk RT, Gail MH, Shi J, Ravel J, Feigelson HS (2015b) Investigation of the association between the fecal microbiota and breast cancer in postmenopausal women: a population-based case-control pilot study. J Natl Cancer Inst 107(8): djv147.

Helicobacter and Cancer Collaborative Group (2001) Gastric cancer and Helicobacter pylori: a combined analysis of 12 case control studies nested within prospective cohorts. Gut 49(3): 347-353.

Hosgood 3rd HD, Sapkota AR, Rothman N, Rohan T, Hu W, Xu J, Vermeulen R, He X, White JR, Wu G, Wei F, Mongodin EF, Lan Q (2014) The potential role of lung microbiota in lung cancer attributed to household coal burning exposures. Environ Mol Mutagen 55(8): 643-651.

Iida N, Dzutsev A, Stewart CA, Smith L, Bouladoux N, Weingarten RA, Molina DA, Salcedo R, Back T, Cramer S, Dai RM, Kiu H, Cardone M, Naik S, Patri AK, Wang E, Marincola FM, Frank KM, Belkaid Y,
Trinchieri G, Goldszmid RS (2013) Commensal bacteria control cancer response to therapy by modulating the tumor microenvironment. Science 342(6161): 967-970.

International Agency for Research on Cancer (1994) IARC Monographs on the Evaluation of Carcinogenic Risks to Humans. Schistosomes, liver flukes and Helicobacter pylori. IARC: Lyon, France.

International Agency for Research on Cancer (2012) IARC Monographs on the Evaluation of Carcinogenic Risks to Humans. Biological Agents. IARC: Lyon, France.

Islami F, Kamangar F (2008) Helicobacter pylori and esophageal cancer risk: a meta-analysis. Cancer Prev Res (Phila) 1(5): 329-338.

Lin WC, Tsai HF, Kuo SH, Wu MS, Lin CW, Hsu PI, Cheng AL, Hsu PN (2010) Translocation of Helicobacter pylori CagA into Human B lymphocytes, the origin of mucosa-associated lymphoid tissue lymphoma. Cancer Res 70(14): 5740-5748.

Meyer MS, Joshipura K, Giovannucci E, Michaud DS (2008) A review of the relationship between tooth loss, periodontal disease, and cancer. Cancer Causes Control 19(9): 895-907.

Michaud DS, Izard J, Wilhelm-Benartzi CS, You DH, Grote VA, Tjonneland A, Dahm CC, Overvad K, Jenab M, Fedirko V, Boutron-Ruault MC, ClavelChapelon F, Racine A, Kaaks R, Boeing H, Foerster J, Trichopoulou A, Lagiou P, Trichopoulos D, Sacerdote C, Sieri S, Palli D, Tumino R, Panico S, Siersema PD, Peeters PH, Lund E, Barricarte A, Huerta JM, MolinaMontes E, Dorronsoro M, Quiros JR, Duell EJ, Ye W, Sund M, Lindkvist B, Johansen D, Khaw KT, Wareham N, Travis RC, Vineis P, Bueno-deMesquita HB, Riboli E (2013) Plasma antibodies to oral bacteria and risk of pancreatic cancer in a large European prospective cohort study. Gut 62(12): $1764-1770$.

Morgan XC, Huttenhower C (2012) Chapter 12: Human microbiome analysis. PLoS Comput Biol 8(12): e1002808.

Murphy G, Michel A, Taylor PR, Albanes D, Weinstein SJ, Virtamo J, Parisi D, Snyder K, Butt J, McGlynn KA, Koshiol J, Pawlita M, Lai GY, Abnet CC, Dawsey SM, Freedman ND (2014) Association of seropositivity to Helicobacter species and biliary tract cancer in the ATBC study. Hepatology 60(6): 1963-1971.

Muto M, Hitomi Y, Ohtsu A, Shimada H, Kashiwase Y, Sasaki H, Yoshida S, Esumi H (2000) Acetaldehyde production by non-pathogenic Neisseria in human oral microflora: implications for carcinogenesis in upper aerodigestive tract. Int J Cancer 88(3): 342-350.

Nagaraja V, Eslick GD (2014) Systematic review with meta-analysis: the relationship between chronic Salmonella typhi carrier status and gallbladder cancer. Aliment Pharmacol Ther 39(8): 745-750.

Nasrollahzadeh D, Malekzadeh R, Ploner A, Shakeri R, Sotoudeh M, Fahimi S, Nasseri-Moghaddam S, Kamangar F, Abnet CC, Winckler B, Islami F, Boffetta P, Brennan P, Dawsey SM, Ye W (2015) Variations of gastric corpus microbiota are associated with early esophageal squamous cell carcinoma and squamous dysplasia. Sci Rep 5: 8820.

Qin J, Li R, Raes J, Arumugam M, Burgdorf KS, Manichanh C, Nielsen T, Pons N, Levenez F, Yamada T, Mende DR, Li J, Xu J, Li S, Li D, Cao J, Wang B, Liang H, Zheng H, Xie Y, Tap J, Lepage P, Bertalan M, Batto JM, Hansen T, Le Paslier D, Linneberg A, Nielsen HB, Pelletier E, Renault P, Sicheritz-Ponten T, Turner K, Zhu H, Yu C, Li S, Jian M, Zhou Y, Li Y, Zhang X, Li S, Qin N, Yang H, Wang J, Brunak S, Dore J, Guarner F, Kristiansen K, Pedersen O, Parkhill J, Weissenbach J, Bork P, Ehrlich SD, Wang J (2010) A human gut microbial gene catalogue established by metagenomic sequencing. Nature 464(7285): 59-65.

Schollkopf C, Melbye M, Munksgaard L, Smedby KE, Rostgaard K, Glimelius B, Chang ET, Roos G, Hansen M, Adami HO, Hjalgrim H (2008) Borrelia infection and risk of non-Hodgkin lymphoma. Blood 111(12): 5524-5529.

Sears CL, Pardoll DM (2011) Perspective: alpha-bugs, their microbial partners, and the link to colon cancer. J Infect Dis 203(3): 306-311.

Segata N, Haake SK, Mannon P, Lemon KP, Waldron L, Gevers D, Huttenhower C, Izard J (2012) Composition of the adult digestive tract bacterial microbiome based on seven mouth surfaces, tonsils, throat and stool samples. Genome Biol 13(6): R42.

Sheflin AM, Whitney AK, Weir TL (2014) Cancer-promoting effects of microbial dysbiosis. Curr Oncol Rep 16(10): 406.

The Human Microbiome Project Consortium (2012) Structure, function and diversity of the healthy human microbiome. Nature 486(7402): 207-214.

Wallace BD, Wang H, Lane KT, Scott JE, Orans J, Koo JS, Venkatesh M, Jobin C, Yeh LA, Mani S, Redinbo MR (2010) Alleviating cancer drug toxicity by inhibiting a bacterial enzyme. Science 330(6005): 831-835. 
Wang L, Ganly I (2014) The oral microbiome and oral cancer. Clin Lab Med 34(4): 711-719.

Weir TL, Manter DK, Sheflin AM, Barnett BA, Heuberger AL, Ryan EP (2013) Stool microbiome and metabolome differences between colorectal cancer patients and healthy adults. PloS one 8(8): e70803.

World Cancer Research Fund/American Institute for Cancer Research (2007) Food, Nutrition, Physical Activity, and the Prevention of Cancer: a Global Perspective. AICR: Washington, DC, USA.

Wotherspoon AC, Doglioni C, Diss TC, Pan L, Moschini A, de Boni M, Isaacson PG (1993) Regression of primary low-grade B-cell gastric lymphoma of mucosa-associated lymphoid tissue type after eradication of Helicobacter pylori. Lancet 342(8871): 575-577.

Wu N, Yang X, Zhang R, Li J, Xiao X, Hu Y, Chen Y, Yang F, Lu N, Wang Z, Luan C, Liu Y, Wang B, Xiang C, Wang Y, Zhao F, Gao GF, Wang S, Li L, Zhang H, Zhu B (2013) Dysbiosis signature of fecal microbiota in colorectal cancer patients. Microb Ecol 66(2): 462-470.

Yang L, Chaudhary N, Baghdadi J, Pei Z (2014) Microbiome in reflux disorders and esophageal adenocarcinoma. Cancer J 20(3): 207-210.

Yu G, Gail MH, Shi J, Klepac-Ceraj V, Paster BJ, Dye BA, Wang GQ, Wei WQ, Fan JH, Qiao YL, Dawsey SM, Freedman ND, Abnet CC (2014) Association between upper digestive tract microbiota and cancerpredisposing states in the esophagus and stomach. Cancer Epidemiol Biomarkers Prev 23(5): 735-741.

Yu J, Feng Q, Wong SH, Zhang D, Liang QY, Qin Y, Tang L, Zhao H, Stenvang J, Li Y, Wang X, Xu X, Chen N, Wu WK, Al-Aama J, Nielsen HJ, Kiilerich P, Jensen BA, Yau TO, Lan Z, Jia H, Li J, Xiao L, Lam TY, Ng SC, Cheng AS, Wong VW, Chan FK, Xu X, Yang H, Madsen L, Datz C, Tilg H, Wang J, Brunner N, Kristiansen K, Arumugam M, Sung JJ, Wang J (2015) Metagenomic analysis of faecal microbiome as a tool towards targeted non-invasive biomarkers for colorectal cancer. Gut; e-pub ahead of print 25 September 2015; doi:10.1136/gutjnl-2015-309800.

Zackular JP, Rogers MA, Ruffin 4th MT, Schloss PD (2014) The human gut microbiome as a screening tool for colorectal cancer. Cancer Prev Res (Phila) 7(11): 1112-1121.

Zeller G, Tap J, Voigt AY, Sunagawa S, Kultima JR, Costea PI, Amiot A, Bohm J, Brunetti F, Habermann N, Hercog R, Koch M, Luciani A, Mende DR, Schneider MA, Schrotz-King P, Tournigand C, Tran Van Nhieu J, Yamada T, Zimmermann J, Benes V, Kloor M, Ulrich CM, von Knebel Doeberitz M, Sobhani I, Bork P (2014) Potential of fecal microbiota for early-stage detection of colorectal cancer. Mol Syst Biol 10: 766 .

Zeng XT, Deng AP, Li C, Xia LY, Niu YM, Leng WD (2013) Periodontal disease and risk of head and neck cancer: a meta-analysis of observational studies. PloS One 8(10): e79017.

Zhou D, Wang JD, Weng MZ, Zhang Y, Wang XF, Gong W, Quan ZW (2013) Infections of Helicobacter spp. in the biliary system are associated with biliary tract cancer: a meta-analysis. Eur J Gastroenterol Hepatol 25(4): 447-454.

Zhu Q, Gao R, Wu W, Qin H (2013) The role of gut microbiota in the pathogenesis of colorectal cancer. Tumour Biol 34(3): $1285-1300$.

(i) (2) (2) This work is licensed under the Creative Commons cc) ${ }_{\text {BY NC }}$ AA Attribution-Non-Commercial-Share Alike 4.0 International License. To view a copy of this license, visit http:// creativecommons.org/licenses/by-nc-sa/4.0/ 